\title{
RursuSpicae
}

Transmission, réception et réécriture de textes, de I'Antiquité au Moyen Âge

$2 \mid 2019$

Le Physiologus. Manuscrits anciens et tradition médiévale

\section{Le dialogue entre l'image et le texte dans le Physiologus de Sofia (Dujčev gr. 297) : le cas de l'echidna}

The Dialogue Between Text and Images in the Physiologus from Sofia (Dujčev gr. 297): the Case of the Echidna

\section{Stavros Lazaris}

\section{OpenEdition}

\section{Journals}

Édition électronique

URL : http://journals.openedition.org/rursuspicae/734

DOI : $10.4000 /$ rursuspicae.734

ISSN : 2557-8839

Éditeur :

Université Nice-Sophia Antipolis, IRHT - Institut de recherche et d'histoire des textes

Référence électronique

Stavros Lazaris, «Le dialogue entre l'image et le texte dans le Physiologus de Sofia (Dujčev gr. 297) : le cas de l'echidna », RursuSpicae [En ligne], 2 | 2019, mis en ligne le 20 décembre 2019, consulté le 29 mars 2021. URL : http://journals.openedition.org/rursuspicae/734 ; DOI : https://doi.org/10.4000/ rursuspicae.734

Ce document a été généré automatiquement le 29 mars 2021

RursuSpicae 


\title{
Le dialogue entre l'image et le texte dans le Physiologus de Sofia (Dujčev gr. 297) : le cas de l'echidna
}

\author{
The Dialogue Between Text and Images in the Physiologus from Sofia (Dujčev \\ gr. 297): the Case of the Echidna
}

Stavros Lazaris

À la mémoire de Xénia Muratova ${ }^{1}$

1 Le Physiologus grec, dont l'auteur reste anonyme, a vu le jour autour du $\mathrm{II}^{\mathrm{e}}$ siècle de notre ère à Alexandrie ${ }^{2}$. Chacun des chapitres est composé de deux parties qui suivent

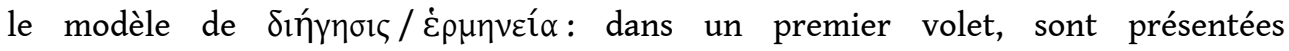

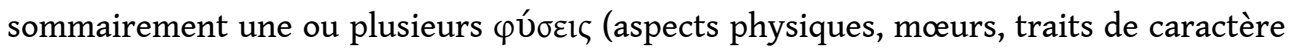
ou encore qualités et défauts supposés) des animaux, végétaux et minéraux traités et, dans un second volet, ces caractéristiques font l'objet d'une interprétation qui reprend

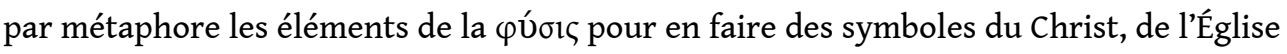
ou encore du Diable. Cette partie herméneutique chrétienne devient donc l'occasion d'une brève leçon de théologie et d'un encouragement moral adressé au lecteur ${ }^{3}$. Le Physiologus a connu un grand essor à Byzance et il a été conservé dans quatre recensions principales. La première, dite chrétienne, est constituée de 48 ou 49 chapitres selon les éditions ${ }^{4}$, voire plus dans certaines sous-familles manuscrites ${ }^{5}$.

Le Physiologus est conservé, toutes recensions confondues, dans 13 manuscrits illustrés ${ }^{6}$. De la première recension, seuls deux manuscrits byzantins enluminés étaient connus jusqu'à dernièrement. Il s'agit de l'Izmir, Euaggelikê Scholê, B-8, pp. 1-137 et du Milano, Biblioteca Ambrosiana, E 16 sup. (Martini-Bassi 273), ff. $1^{\mathrm{r}}-41^{\mathrm{r}}$. Le premier a été copié, selon plusieurs spécialistes, au $\mathrm{XI}^{\mathrm{e}}$ siècle tandis que le second est du $\mathrm{XII}^{\mathrm{e}}$ siècle. Le manuscrit de Smyrne a été détruit en 1922 dans un incendie, mais, fort heureusement, sont conservées des reproductions en noir et blanc de la majeure partie de ses illustrations ${ }^{7}$. Un troisième témoin a été trouvé en 1999 : il s'agit du Sofija, Naučen Centăr 
za Slavjano-Vizantijski Proučvanija «Ivan Dujčev », D. gr. 297, ff. 163 ${ }^{\mathrm{r}}-200,1^{\mathrm{v}}$, du début de la seconde moitié du XVI ${ }^{\mathrm{e}}$ siècle ${ }^{8}$.

3 Le texte du Physiologus de Sofia présente la particularité d'être composé de 51 chapitres au lieu des 48 ou 49 (suivant l'édition critique de la première recension, voir supra, n. 4). En effet, y ont été ajoutés trois chapitres : $\pi \varepsilon p \grave{i} \theta \dot{u}<v>v o v$ (sur le thon), ff. $184^{\mathrm{v}}-185^{\mathrm{r}}$;

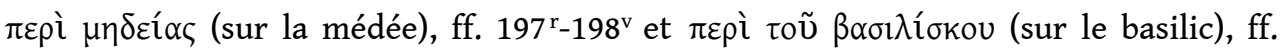
$198^{\mathrm{v}}-200^{\mathrm{v}}$. Ces chapitres se retrouvent dans quelques autres manuscrits, sans toutefois qu'ils soient illustrés'. Les images de ces chapitres dans le codex de Sofia constituent alors un unicum.

de ce manuscrit est orné de 55 miniatures accompagnant 47 chapitres ${ }^{10}$. Toutes les images sont placées dans le corps du texte et cette mise en page requière une collaboration étroite entre copiste et miniaturiste, à moins qu'il ne s'agisse de la même personne. Ce choix facilite la lecture en créant un découpage rythmique de la page dans l'ordre suivant : titre/texte du premier volet/image/texte du second volet ${ }^{11}$. L'attention du lecteur est alors focalisée sur l'essentiel et les choix iconographiques renforcent ceci. En effet, le miniaturiste de ce manuscrit, dans la majorité des cas, ne représente que le sujet du chapitre, faisant abstraction de l'historia textuelle. Ici, à la différence de plusieurs autres codex illustrés, les animaux, végétaux et minéraux ne sont pas des éléments constitutifs d'une composition, mais l'objet unique. Soulignons également l'absence de décor. Cette volonté d'aider le lecteur à se concentrer sur l'essentiel se révèle par l'élimination de tout détail inutile à la compréhension de la miniature. Ainsi, non seulement ce manuscrit vient enrichir la petite liste de codex illustrés de la première recension, mais aussi il présente certaines originalités qui en font un témoin important dans l'étude de l'illustration du Physiologus grec. Ses qualités m'ont incité à lui consacrer plusieurs études ${ }^{12}$.

Dans la présente contribution, je me focaliserai sur une variante iconographique entre ce manuscrit et les autres témoins de la première et de la deuxième recensions ${ }^{13}$. Je m'intéresserai également aux rapports entre le texte et l'image dans les différents codex examinés et les renseignements que l'on peut en tirer pour l'identification de l'espèce analysée dans le cadre de cette étude : l'echidna ${ }^{14}$.

6 Avant de passer en revue les rapports de l'image et du texte qui composent ce chapitre, exposons rapidement le cadre méthodologique suivi. Pour le cas de figure analysé ciaprès, je propose, outre la reproduction et la description de l'image de l'echidna, une transcription diplomatique du texte du manuscrit. Le but de cette dernière est de révéler au lecteur moderne les particularités du texte spécifique de ce codex, tel que le miniaturiste, puis les lecteurs successifs l'avaient devant leurs yeux. Privilégier les variantes du texte et l'altérer le moins possible, tel a donc été mon choix, qui plaide en faveur d'une orientation ad manuscriptum de sorte que l'on puisse comprendre autant que possible la construction du sens par le lecteur médiéval ${ }^{15}$. En plus des rapprochements avec les miniatures des deux autres manuscrits illustrés du Physiologus de la première recension, je procèderai aussi à des parallèles avec deux codex contenant le texte de la deuxième recension, ainsi qu'avec un représentant de la traduction latine par l'intermédiaire du plus ancien témoin conservé, le fameux Physiologus Bernensis, dont les réminiscences hellénistiques ne font aucun doute ${ }^{16}$. Le Physiologus de Sofia ayant été confectionné dans un milieu grec et n'ayant subi aucune influence iconographique occidentale directe, je n'ai pas cru utile, malgré sa date tardive, de procéder à des comparaisons avec des manuscrits occidentaux tardifs. Plus 
précisément, les quelques rapprochements menés n'ont donné aucun résultat probant pour les objectifs de la présente étude.

\section{Le Sofija, Naučen Centăr za Slavjano-Vizantijski Proučvanija « Ivan Dujčev », D. gr. 297}

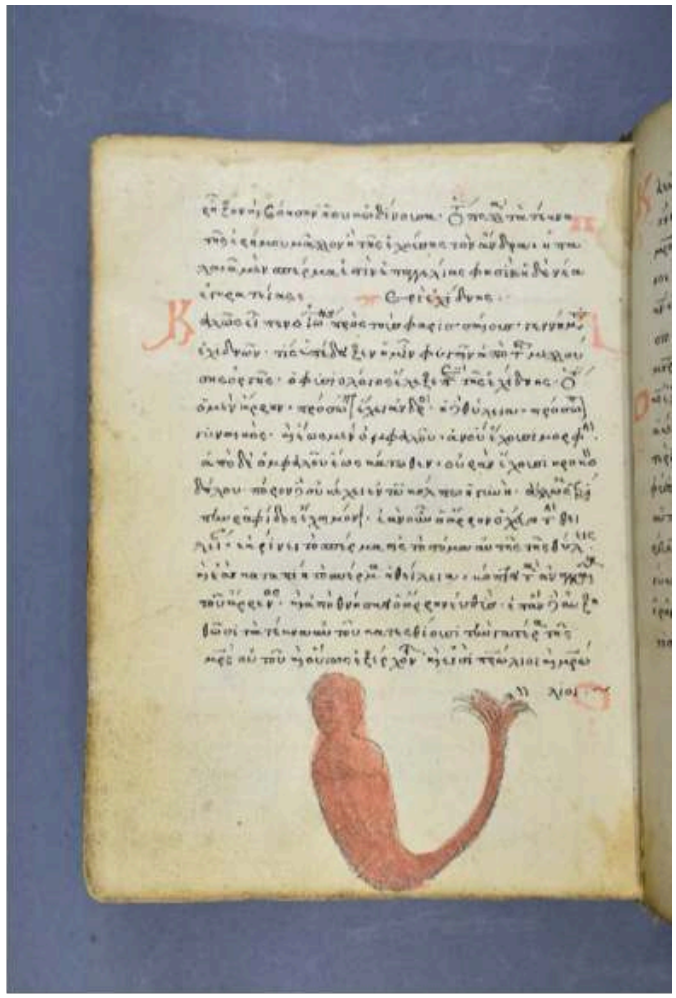

Fig. 1 : Sofija, Naučen Centăr za Slavjano-Vizantijski Proučvanija «Ivan Dujčev », D. gr. 297, f. 169v

7 La miniature (f. $169^{\mathrm{v}}, 70$ x $60 \mathrm{~mm}$.) consiste en la représentation d'un être hybride, qui est composé de deux parties : un buste anthropomorphe (le thorax et l'abdomen sont suggérés par quelques coups de crayon) et un corps/queue qui se termine comme la nageoire caudale (type homocerque) des poissons ${ }^{17}$. Une tête aux cheveux courts prolonge le buste sans bras, sur lequel furent tracés des pectoraux et des abdominaux. De biais, ces deux parties forment un angle saillant en forme de V. L'espace du nombril jusqu'à la nageoire est hachuré. L'animal est peint à grands traits rouges (fig. 1). 
$\Pi \varepsilon \rho \grave{~} \chi^{\prime} \delta ́ \delta \vee \eta \varsigma^{18}$

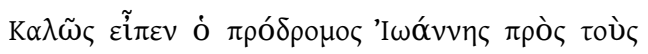

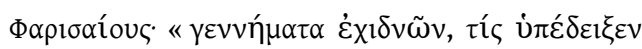

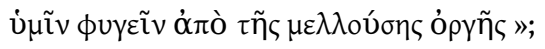

O Фuఠı

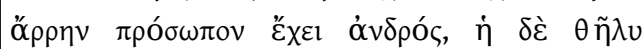

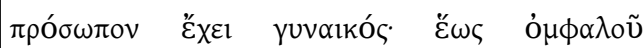

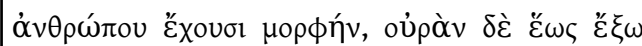

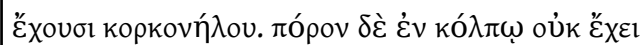

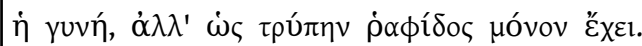

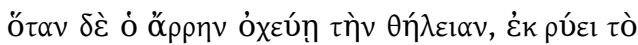

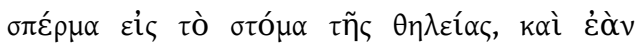

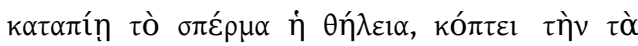

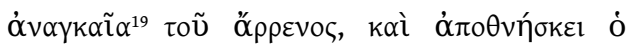

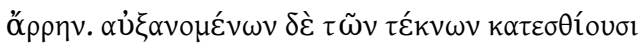

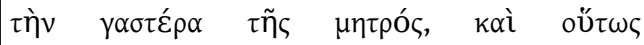

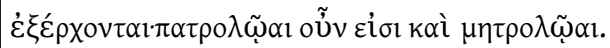

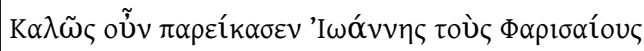

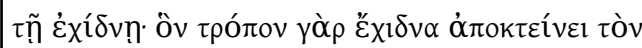

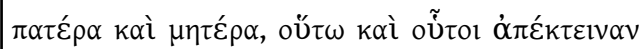

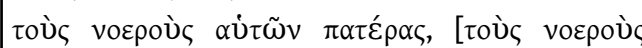

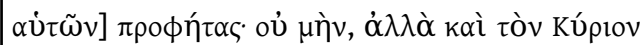

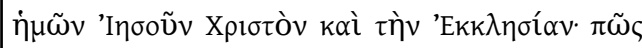

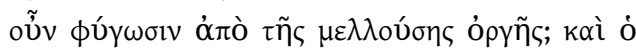

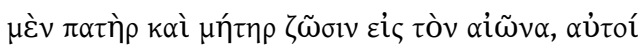

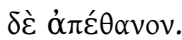

\section{Sur l'echidna ${ }^{20}$}

C'est à juste titre que Jean le Précurseur a dit aux Pharisiens: «Engeance de vipère ${ }^{21}$, qui vous a montré le moyen d'échapper à la colère qui vient?»

Le Physiologue a dit ceci à propos de l'echidna: le mâle a le visage d'un homme, la femelle, le visage d'une femme; jusqu'au nombril, ils ont une apparence humaine, mais ensuite, la queue d'un crocodile. La femelle n'a pas de passage conduisant à l'utérus : elle a seulement comme un trou d'aiguille. Lorsque le mâle s'accouple à la femelle, il émet sa semence dans la bouche de la femelle; et une fois que la femelle a avalé la semence elle coupe les parties génitales du mâle et ce dernier meurt. Lorsque ses petits grandissent, ils dévorent le ventre de la mère et c'est ainsi qu'ils viennent au jour. Ainsi sontils parricides et matricides ${ }^{22}$.

C'est donc à juste titre que Jean a comparé les Pharisiens à la vipère. Car, de la même façon que la vipère tue ses père et mère, ils ont, eux, tué leurs parents spirituels, les Prophètes; et jusqu'à notre Seigneur, Jésus Christ, et l'Église. Comment, dès lors, échapperaient-ils à «la colère qui vient »? Le père et la mère vivront pour l'éternité, mais eux, ils sont morts.

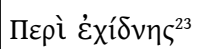

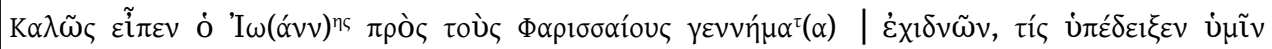

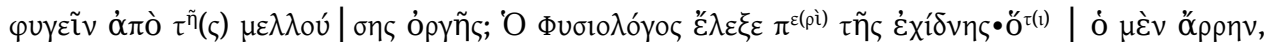

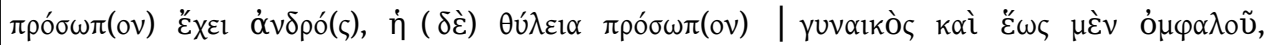

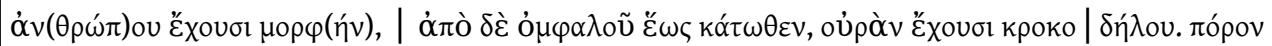

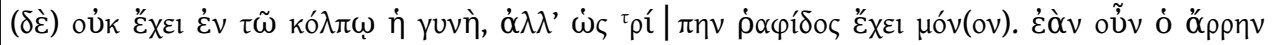

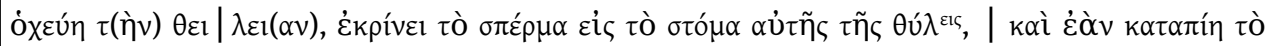

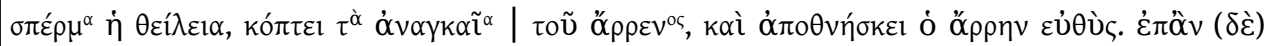

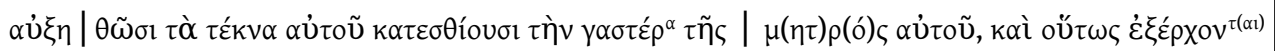

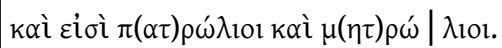

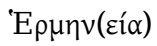

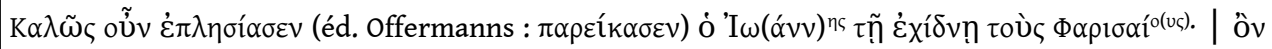

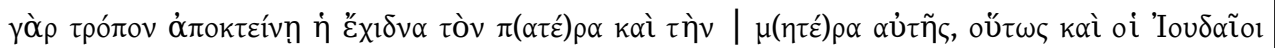

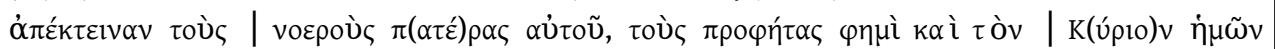

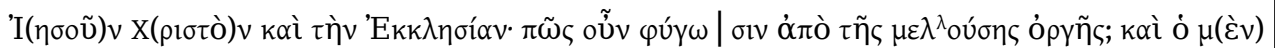

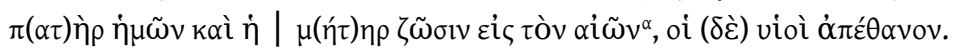

8 Correspondance texte-image : le miniaturiste du Physiologus de Sofia semble retenir la toute première phrase du texte de la partie naturaliste, où il est question de l'echidna mâle qui a le visage d'un homme. Il est précisé que cet être a une apparence humaine 
jusqu'au nombril, puis la queue d'un crocodile. L'echidna femelle, le comportement sexuel, ainsi que l'enfantement n'ont pas retenu son attention.

\section{Les autres manuscrits illustrés}

Physiologus graecus (première recension). Dans le Physiologus de Smyrne (Izmir, Euaggelikê Scholê, B-8, p. 40), le miniaturiste représente deux êtres hybrides dont la partie centrale est composée, de la tête jusqu'au nombril, d'un corps humain féminin. La partie inférieure de leur corps est constituée d'une queue ondoyante qui fait penser à celle d'un serpent ou d'un crocodile. Soulignons également que chaque être est pourvu d'une paire de pattes de rapace (seulement trois sur les quatre doigts sont apparents) aux griffes saillantes. De celui de gauche, au niveau des côtes, deux autres corps humains, nus également, sortent (fig. 2). Il s'agirait ici d'une scène qui se rapporte au tout dernier passage textuel du volet naturaliste, dans lequel il est précisé que les petits de l'echidna, lorsqu'ils grandissent dans le ventre de leur mère, le dévorent et c'est ainsi qu'ils

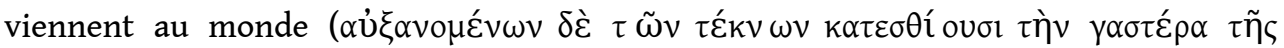

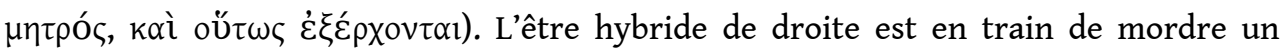
corps masculin au niveau des parties génitales. La miniature ferait référence ici au

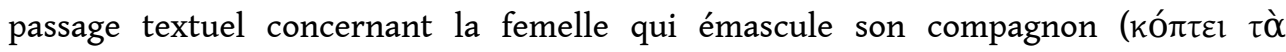

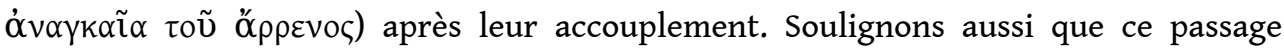
textuel devance le précédent dans le Physiologus, tandis que l'image, si on part du principe que la miniature doit être lue de gauche à droite, les présente dans l'ordre inverse.

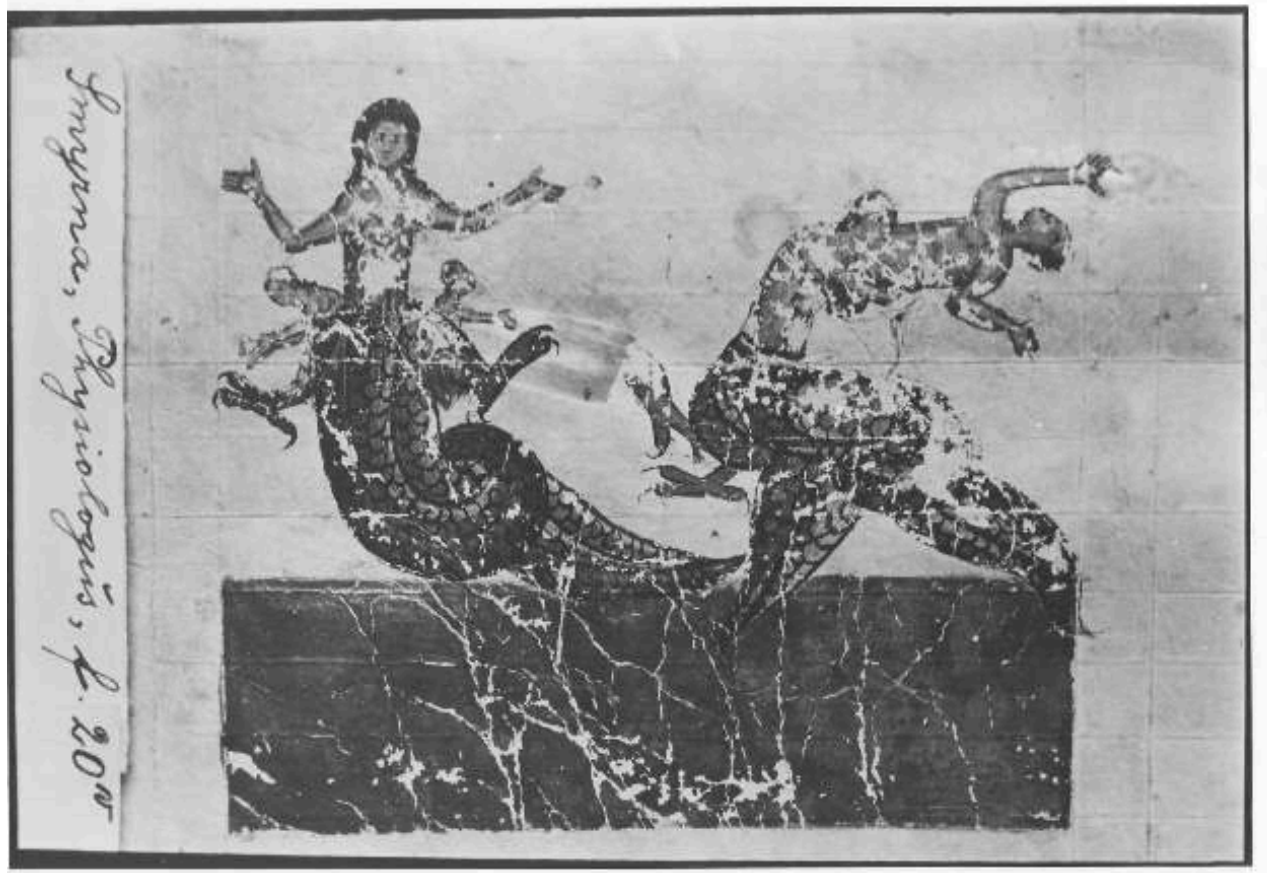

Fig. 2 : Izmir, Euaggelikê Scholê, B-8, p. 40 


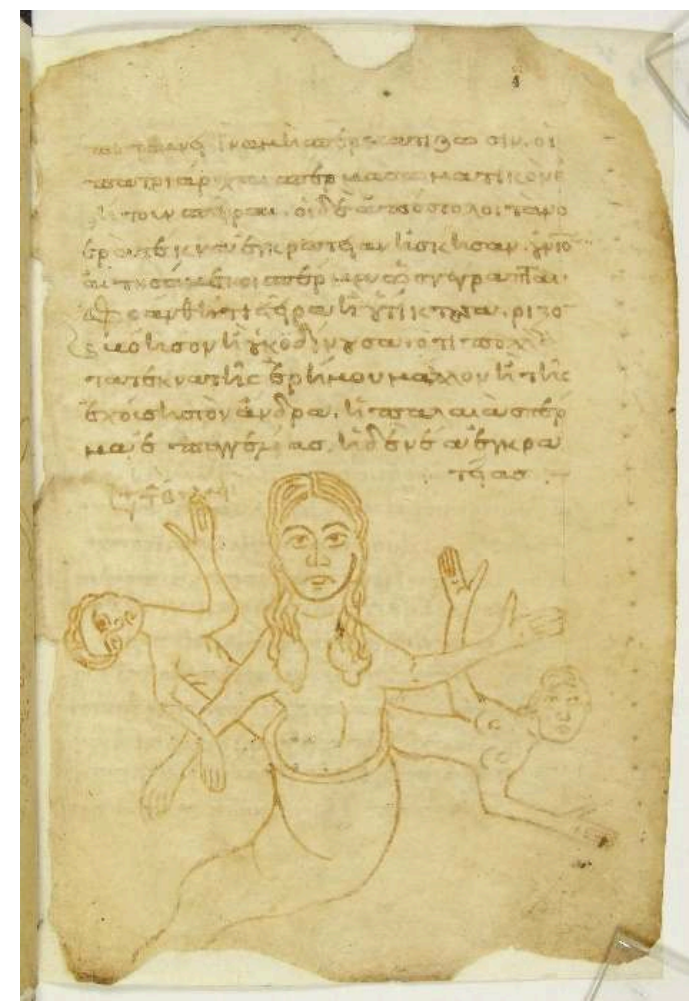

Fig. 3 : Milano, Biblioteca Ambrosiana, E 16 sup. [Martini-Bassi 273], f. 4 r Ambrosiana, E 16 sup. [Martini-Bassi 273], f. $4^{\text {r) }}$ est aussi figuré un être hybride dont la partie supérieure est composée, de la tête jusqu'au nombril, d'un corps humain féminin à longs cheveux et torse nu et, à la place de la partie inférieure, d'un corps qui ressemble de nouveau à celui d'un serpent ou à une queue ondulée de crocodile. Deux autres corps humains, nus également, un masculin (à gauche de l'image) et un féminin (à droite) semblent sortir des côtés de cet être central. Il s'agirait de nouveau ici d'une scène se référant aux petits qui dévorent le ventre de leur mère pour sortir (fig. 3).

Physiologus graecus (recensions mixtes ${ }^{24}$ ). Dans les autres manuscrits où ce chapitre est illustré, le texte provient de la deuxième recension et il n'est pas tout à fait identique à celui de la première, sans toutefois que le contenu soit altéré en profondeur. En effet, ici aussi il est question d'un être hybride mi-homme/mi-crocodile. De même, il est précisé que quand l'echidna mâle et l'echidna femelle s'accouplent, cette dernière coupe les parties génitales du mâle et tombe enceinte tandis que son compagnon meurt sur le coup. Enfin, le rédacteur de cette recension reprend le passage où est souligné la façon dont les petits déchirent de l'intérieur le ventre de leur mère pour en sortir. Ce chapitre n'a pas été repris dans tous les manuscrits ${ }^{25}$ des recensions mixtes et dans certains codex, à l'instar du Vat. gr. 695 (f. 198 ${ }^{\mathrm{r}}$ ), il a été copié, mais sans recevoir un équivalent visuel.

Quant aux manuscrits illustrés, ils n'ont pas tous la même iconographie. Ainsi, dans le Vat. Ottob. gr. 354 (f. $45^{\mathrm{r}}$ ), nous avons, sur le registre supérieur, deux êtres hybrides (corps d'un mâle humain au torse nu et au corps de serpent ou de crocodile à la place de la partie inférieure $)^{26}$ et, au registre inférieur, un être hybride de sexe féminin avec la tête d'un petit en train de sortir au niveau de son bas ventre. Les trois êtres possèdent des bras avec des mains et ils sont ailés. Remarquons que leurs doigts sont au 
nombre de trois au lieu de cinq. Autour des doigts de l'être du haut, apparaît même une sorte de fine membrane qui les relie, un peu comme la palmure des amphibiens (fig. 4). Par contre, dans le Vindob. Phil. gr. 290 (f. $22^{\text {r }}$ ), l'iconographie diffère. Ici, nous voyons trois créatures, mi-humains/mi-animaux. La figure de gauche représente un hybride avec, pour sa partie supérieure, un homme vêtu d'une robe rouge et pour sa partie inférieure, celle d'un lion. Lui fait face un être quasi identique, mais, cette fois-ci, de sexe féminin. Ce dernier pointe de l'index l'autre être hybride et un dialogue semble être instauré entre les deux. Enfin, un troisième hybride est peint à droite de l'image. Il est mi-mâle/mi-crocodile ou dragon. En effet, sa partie supérieure est celle d'un homme habillé avec une robe rouge et sa partie inférieure est composée d'une longue queue verte ondoyante et d'une paire de pattes. La présence de celles-ci, aux griffes apparentes, incite à exclure la représentation d'un serpent, même si quelques iconographies de serpents à pattes existent (fig. 5).

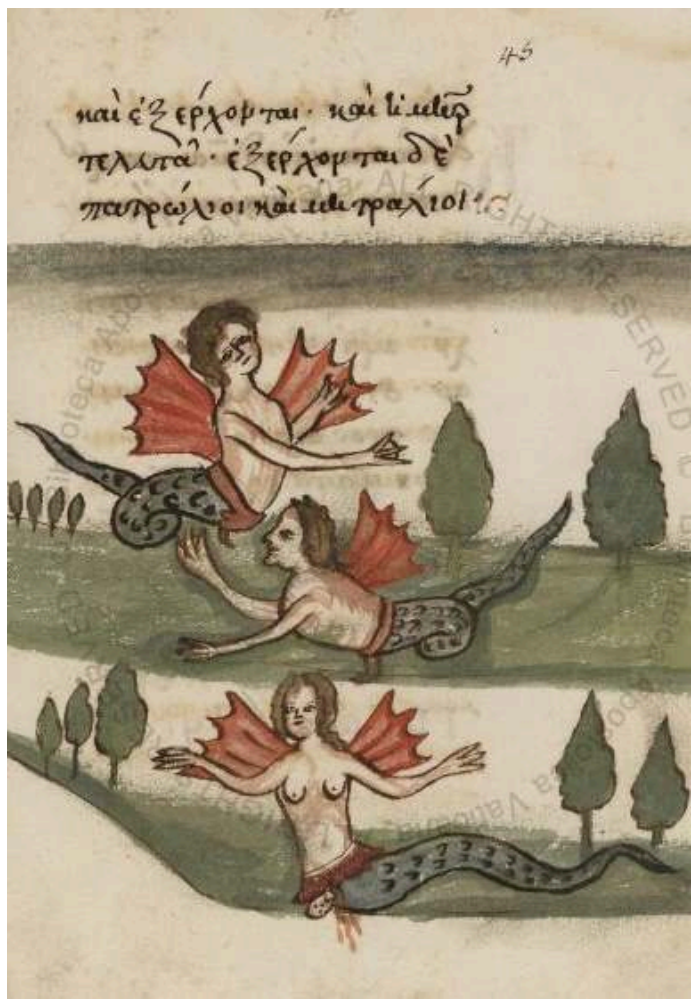

Fig. 4 : Vaticano, Biblioteca Apostolica Vaticana, Vat. Ottob. gr. 354, f. $45^{r}$ 


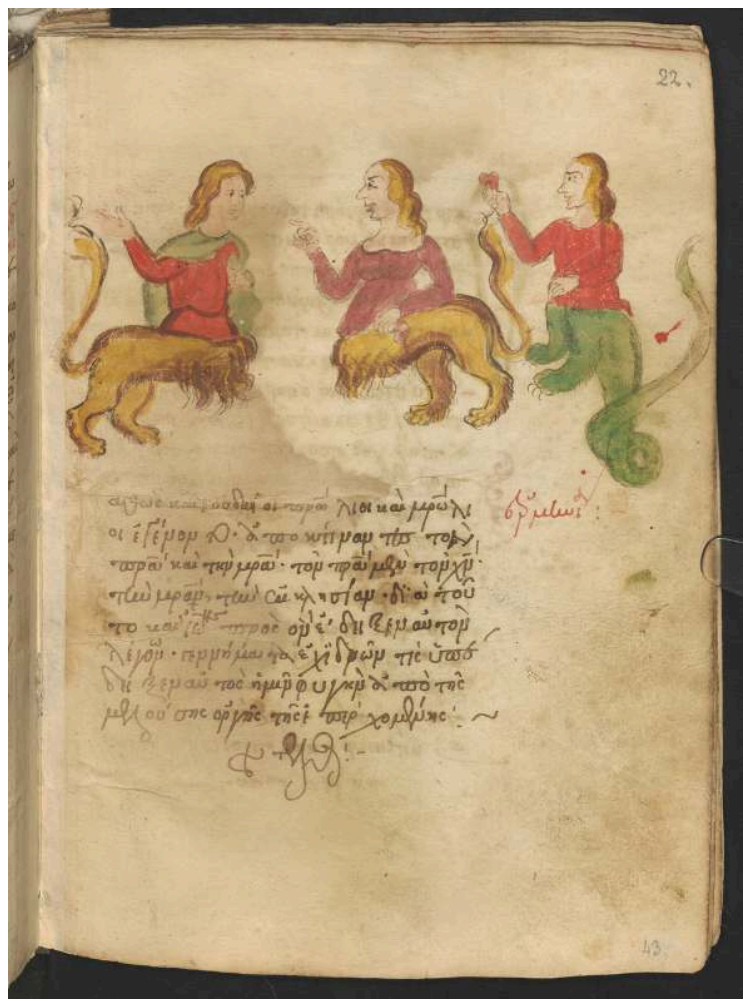

Fig. 5 : Wien, Österreichische Nationalbibliothek, Phil. gr. 290, f. 22

Physiologus latinus. Le miniaturiste du Physiologus latin de Berne (Bern, Burgerbibliothek, $318, \mathrm{f} .11^{\mathrm{r}}$ ) a représenté face à face deux êtres hybrides, un mâle et une femelle ${ }^{27}$, avec la partie inférieure de leur corps qui ressemble à celle d'un poisson ${ }^{28}$ pour le mâle et à celle d'un serpent ou d'un crocodile pour la femelle (figs. 6-7).

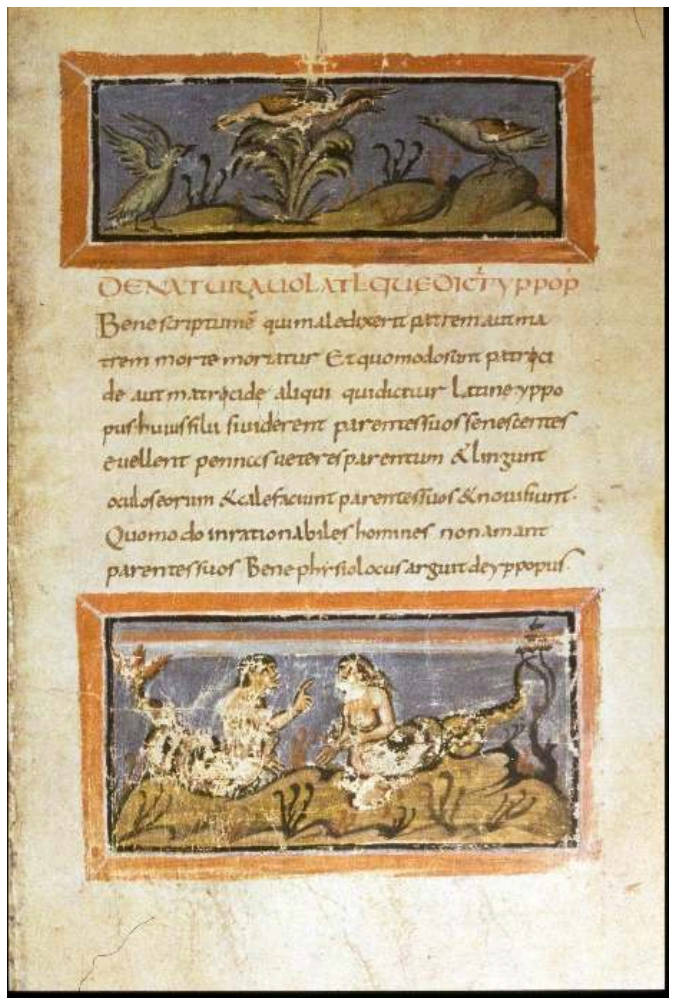

Fig. 6 : Bern, Burgerbibliothek, 318, f. $11^{r}$ (miniature) 


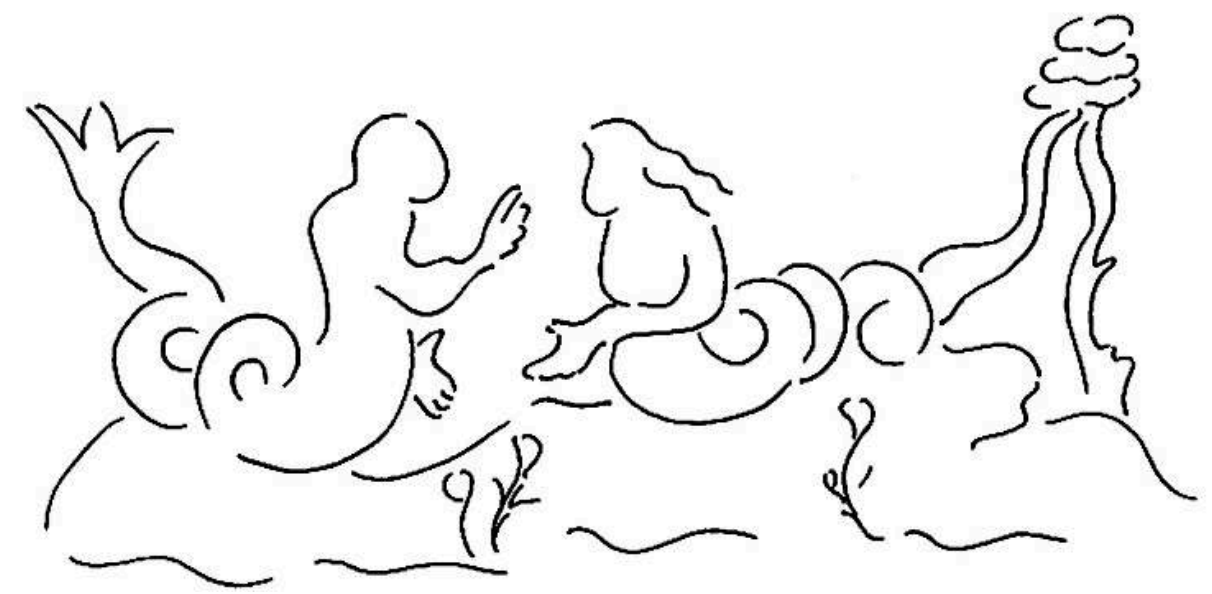

Fig. 7 : Bern, Burgerbibliothek, 318, f. $11^{r}$ (dessin)

On peut résumer les différentes manières de représenter l'echidna comme suit :

\begin{tabular}{|c|c|c|c|c|c|}
\hline Sofia & Smyrne & Milan & Vatican & Vienne & Berne \\
\hline $\begin{array}{l}\text { Un seul } \\
\text { être } \\
\text { hybride : } \\
\text { mi-mâle/ } \\
\text { mi- } \\
\text { poisson. }\end{array}$ & $\begin{array}{l}\text { Deux êtres } \\
\text { hybrides : mi- } \\
\text { femelle/mi- } \\
\text { serpent ou } \\
\text { crocodile. } \\
\text { Celui de } \\
\text { gauche avec } \\
\text { ses petits, } \\
\text { celui de droite } \\
\text { en train de } \\
\text { morde un être } \\
\text { mâle. }\end{array}$ & $\begin{array}{l}\text { Un etre } \\
\text { hybride : mi- } \\
\text { femelle/mi- } \\
\text { serpent ou } \\
\text { crocodile } \\
\text { avec ses } \\
\text { petits. }\end{array}$ & 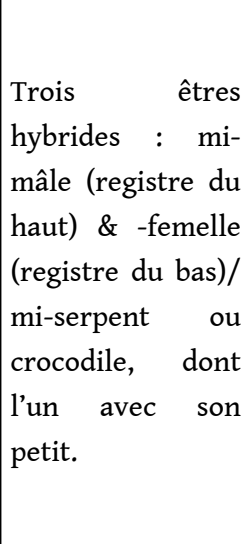 & $\begin{array}{l}\text { Trois êtres } \\
\text { hybrides : un mi- } \\
\text { mâle/mi-lion et } \\
\text { un autre mi- } \\
\text { femelle/mi-lion; } \\
\text { un troisième être } \\
\text { mi-mâle/mi- } \\
\text { crocodile ou } \\
\text { dragon. }\end{array}$ & 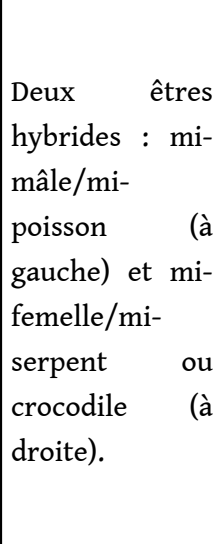 \\
\hline
\end{tabular}

\section{Dissemblances et ressemblances}

Après ce tour d'horizon des différentes manières de "donner à voir " au lecteur l'echidna dans les manuscrits du Physiologus, essayons d'analyser les désaccords qui caractérisent la façon de procéder du codex de Sofia par rapport à ses confrères et les renseignements que l'on peut en tirer. Tout d'abord, il se distingue des autres en se limitant au sujet principal du chapitre, sans chercher à mettre en scène les mœurs de l'echidna (manuscrits de Smyrne, Milan et Vatican) ou même une quelconque relation entre le mâle et la femelle (codex de Vienne [deux hybrides de gauche] et de Berne). Ceci, comme cela a été relevé, est une des caractéristiques propres des miniatures de ce manuscrit. Notre imagier cherche avant tout à produire des « portraits » animaliers.

Remarquons également que la partie inférieure du corps de l'echidna ressemble à celle d'une queue de poisson. Le miniaturiste du Physiologus de Sofia a choisi une représentation plus proche de la sirène telle que nous la connaissons dans 
l'iconographie occidentale ${ }^{29}$. Soulignons toutefois que dans le manuscrit de Sofia l'être semble être de sexe masculin. Cette dernière caractéristique fait d'ailleurs penser à Triton, sauf que la queue dans le codex de Sofia n'est pas particulièrement longue, comme c'est habituellement le cas dans l'iconographie de ce dieu marin.

À l'opposé, dans les autres manuscrits grecs examinés, l'echidna est figurée avec une queue de serpent ou de crocodile, voire de dragon. Représenter l'echidna en tant qu'être hybride, avec la partie supérieure (tête, torse, bras) comme celle d'une femme et la partie inférieure comme celle d'un serpent, correspond parfaitement à la description de l'Echidna dans la Théogonie d'Hésiode (295-305) ${ }^{30}$. S'il s'agit donc bien d'une queue de serpent, les miniaturistes des manuscrits grecs de Smyrne, de Milan et du Vatican seraient fidèles à l'iconographique traditionnelle, sans prendre en considération, au mot près, le texte du Physiologus où il est précisé que la partie inférieure de cet être

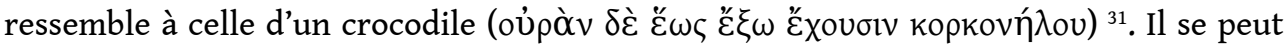
aussi que nous soyons en présence d'une correction de la part de ces miniaturistes d'une variante du texte du Physiologus perçue comme une erreur. Cette hypothèse me semble toutefois peu probable puisque les miniaturistes des manuscrits cités ci-dessus proposent la même iconographie sans pourtant que l'on puisse déceler un lien quelconque entre eux (même modèle, séjour dans une même bibliothèque ...). Il se peut aussi que la partie inférieure de ces êtres hybrides soit bien celle d'un crocodile. Cela ne doit pas être dû à une méconnaissance du crocodile ${ }^{32}$, mais plutôt à une assimilation au Moyen Âge du crocodile au dragon/serpent ${ }^{33}$. Parfois cet animal est en effet représenté, tant en Occident qu'en Orient, avec une queue qui ressemble à celle d'un serpent ${ }^{34}$. Hormis la différence entre la partie inférieure de la représentation dans ces quatre manuscrits grecs, alors que l'echidna est composée dans la partie supérieure d'un corps de femme ${ }^{35}$, dans le codex de Sofia, c'est un mâle qui compose cette partie.

Comment peut-on expliquer ces différences iconographiques entre le Physiologus de Sofia et celle des autres codex grecs? Est-ce que les particularités constatées dans le codex de Sofia font référence à une autre source?

Trois auteurs antérieurs et/ou contemporains du rédacteur du Physiologus parlent de l'echidna. Il s'agit de Nicandre dans ses Theriaka (v. 128-134 et 209-258), de Pline dans son Historia naturalis $(\mathrm{X}, 169)$ et d'Élien dans son De natura animalium (I, 24). C'est à Nicandre que l'on doit l'information concernant les petits de l'echidna qui dévorent la mince paroi du ventre maternel pour sortir. Selon le poète, en faisant ainsi, ils vengent leur père mort par leur mère pendant leurs ébats charnels. Pline nous informe que le mâle introduit sa tête dans la gueule de la femelle qui la ronge dans la jouissance voluptueuse. Élien, enfin, reprend la mort du mâle par la femelle. Selon lui, cette dernière enserre le cou de son compagnon et elle le décapite, en lui tranchant net la tête. À part Nicandre, qui décrit la morphologie de l'echidna, et dont la description ne laisse aucun doute quant à l'identification de cet animal avec un serpent, en l'occurrence la vipère, les autres auteurs ne sont pas prolifiques pour ce qui est de sa description morphologique. Cela dit, rien dans tous ces écrits ne laisse supposer qu'ils sont à l'origine de la différence iconographique relevée dans le Physiologus de Sofia. De toute façon, dans ces ouvrages, il n'est point question d'un être hybride et tout laisse croire que, non seulement Nicandre, mais les deux autres aussi considéraient l'echidna comme étant un serpent.

De même, il faut exclure une influence depuis un autre passage du De natura animalium

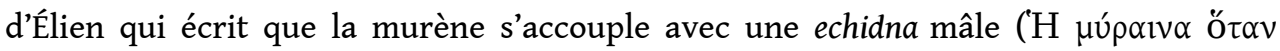




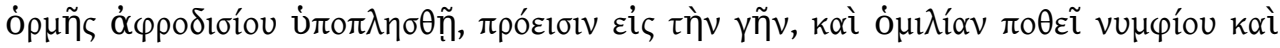

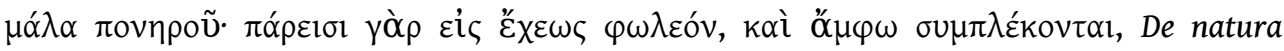
animalium, I, 50, éd. García Valdés, Llera Fueyo and Rodríguez-Noriega Guillén, "Claudius Aelianus de natura animalium"). Ajoutons aussi que, dans l'iconographie traditionnelle, les deux animaux (echidna et murène) sont représentés face à face et l'echidna est clairement montré comme étant un serpent (cf. Paris, BNF, gr. 923, f. $199^{\mathrm{r}}$ [fig. 8] ; Paris, BNF, gr. 2737, f. $98^{v}$ [fig. 9]), ce qui renforce par ailleurs mes précédentes conclusions sur l'identification de l'echidna avec un serpent chez les auteurs présentés ci-dessus.

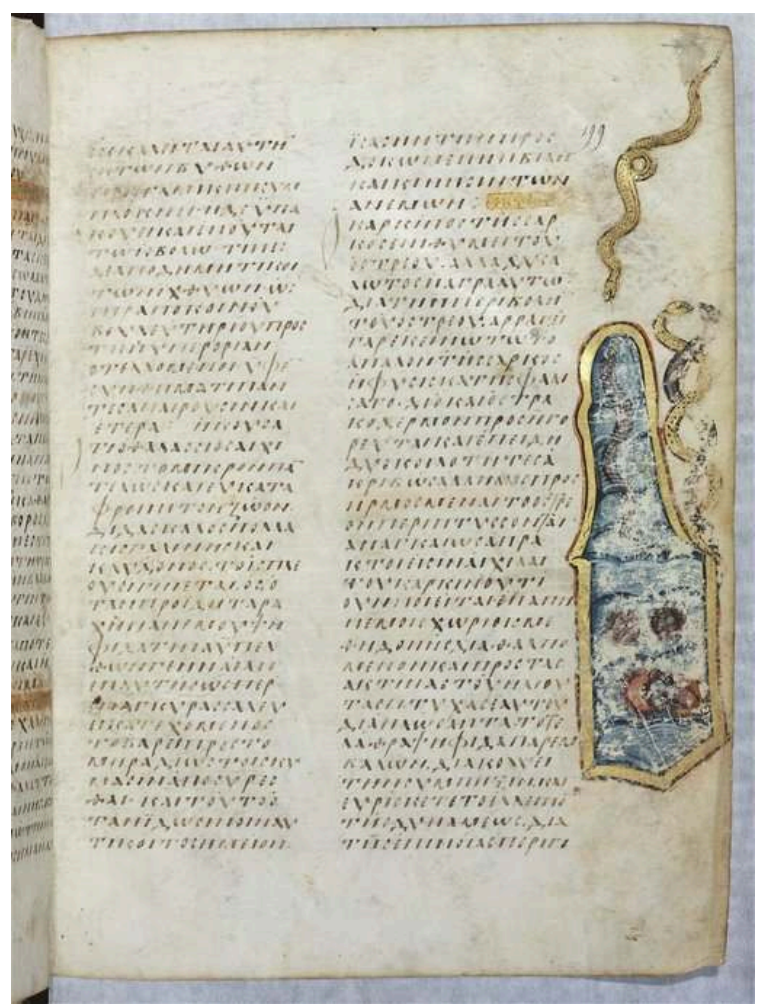

Fig. 8 : Paris, Bibliothèque nationale de France, gr. 923, f. 199r 


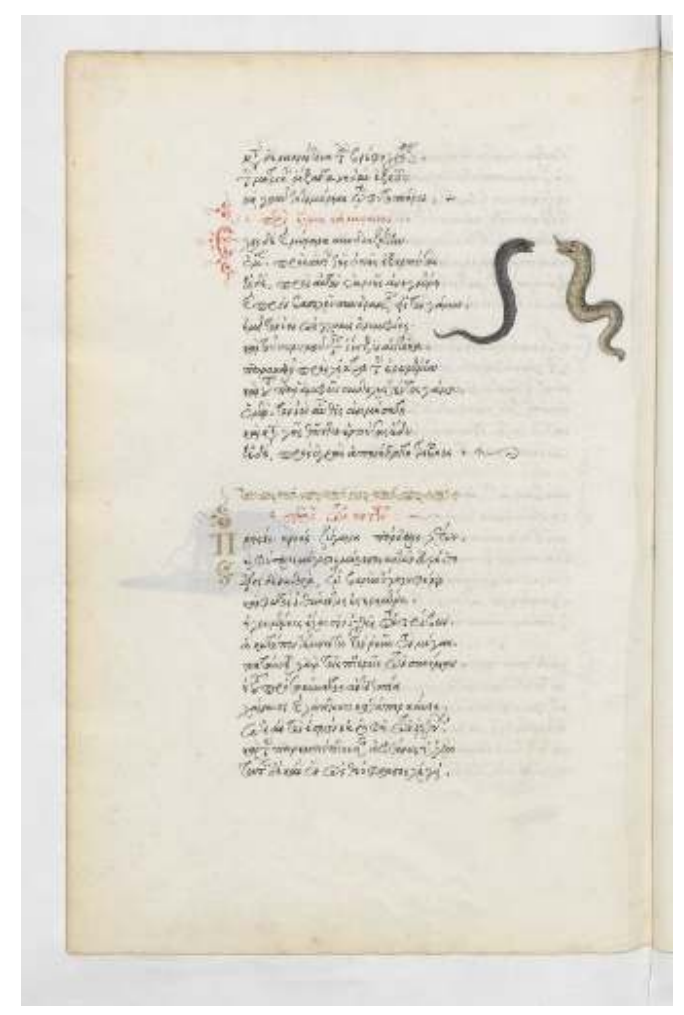

Fig. 9 : Paris, Bibliothèque nationale de France, gr. 2737, f. $98^{\vee}$

Une autre hypothèse serait que le miniaturiste du Physiologus de Sofia a voulu

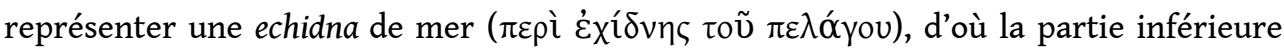
qui ressemble à la queue d'un poisson. Notons toutefois que cet animal apparaît non pas dans la première recension du Physiologus, comme c'est le cas dans ce manuscrit, mais dans la quatrième ${ }^{36}$. Une telle hypothèse suggèrerait alors que le miniaturiste connaissait le texte de cette recension, ce qui ne peut pas être exclu, mais indémontrable dans l'état actuel de nos connaissances.

Enfin, une troisième hypothèse conduirait à voir derrière cette image de l'echidna mimâle/mi-poisson une contamination avec un type iconographique de l'echidna attesté par ailleurs. En effet, le miniaturiste du Physiologus de Berne a par exemple aussi dessiné l'un des deux êtres du chapitre sur « La natura della vipera » avec une queue de poisson (figs. 5 et 6 , hybride de gauche). Pourtant, le texte de ce manuscrit suit l'original grec, où il est question de crocodile et non de serpent ou de poisson. Nous lisons en effet au folio $11^{\mathrm{v}}$ : Physiologus monet de uipera quoniam faciem habet uiri, femina mulieris, usque ad umbiculum, et usque ad caudam corcodrillo habet figuram ${ }^{37}$. Une différence demeure entre les deux représentations au niveau de la forme de la queue : celle du manuscrit de Sofia fait penser à une nageoire caudale de type homocerque, tandis que celle du manuscrit de Berne ressemble plutôt à un trident, l'attribut de Poséidon. Cette même forme se rencontre également dans des manuscrits grecs pour représenter des animaux aquatiques, à l'instar du Venezia, Biblioteca Nazionale Marciana, Marc. gr. Z. 479 (coll. 881), f. $3^{\text {r }}$, fig. 10 (animal à droite dans le registre inférieur) ${ }^{38}$. Toutefois, dans des copies postérieures de ce manuscrit (cf. Paris, BNF, gr. 2737, f. $3^{\text {r }}$, fig. 11), la forme tridentée de la queue changera en une forme quasi-identique à celle de l'echidna du Physiologus de Sofia, qui est de la même époque (XVI ${ }^{\mathrm{e}}$ s.) que le Paris. gr. 2737. Cette 
différence dans le rendu de la forme n'a donc rien de significatif. Ajoutons enfin que dans le codex de Berne c'est aussi l'être mâle qui a une queue de poisson.

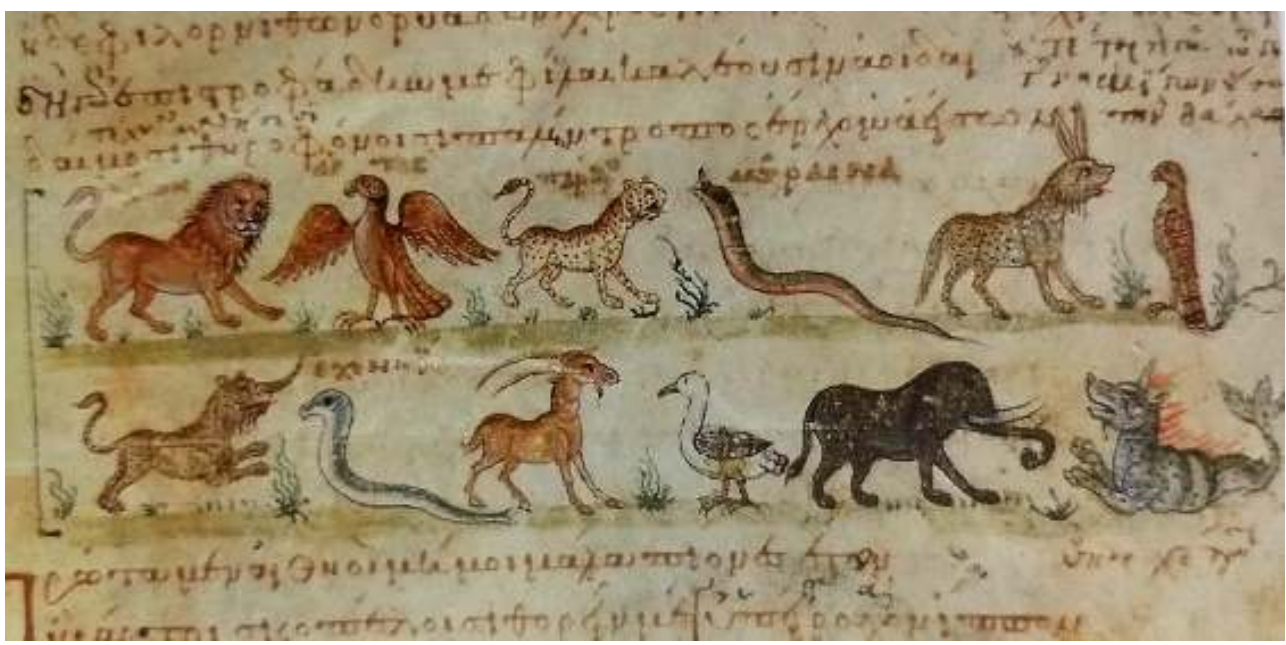

Fig. 10 : Venezia, Biblioteca Nazionale Marciana, Marc. gr. Z. 479 (coll. 881), f. 3

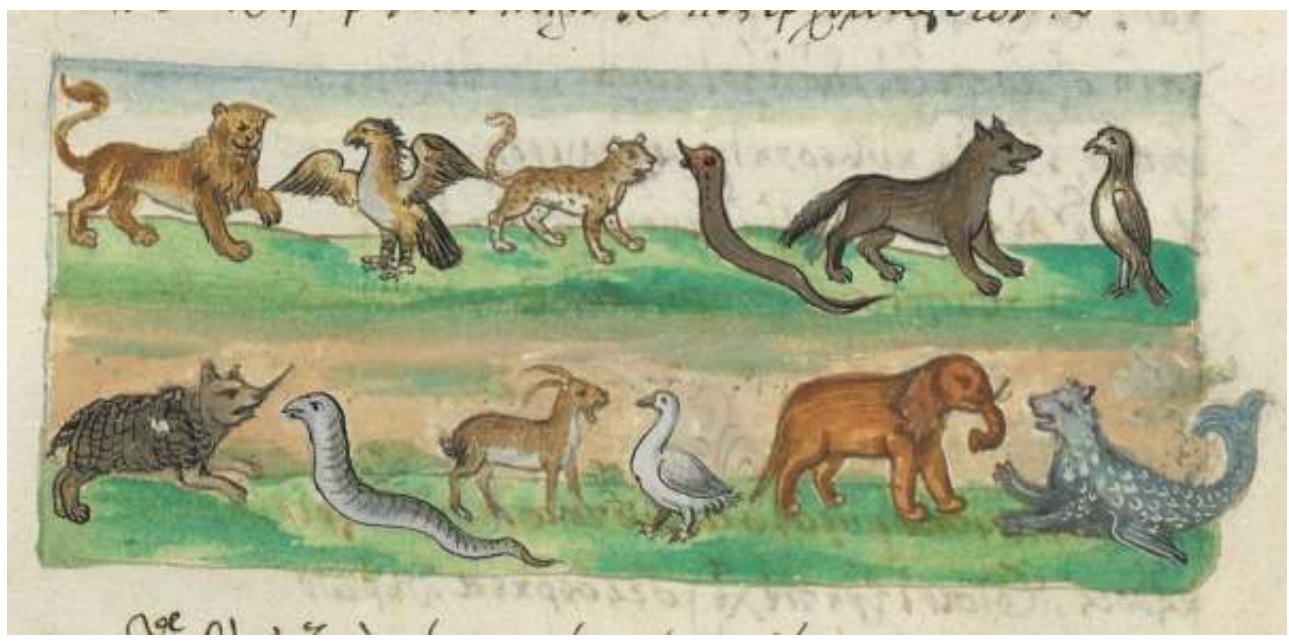

Fig. 11 : Paris, Bibliothèque nationale de France, gr. 2737, f. $3^{r}$

Après avoir évalué les différentes hypothèses (philologiques et visuelles) pour expliquer les dissemblances iconographiques entre la représentation du Physiologus de Sofia et celles des autres manuscrits grecs, deux demeurent à mes yeux plausibles : soit le miniaturiste de Sofia connaissait le texte d'un Physiologus de la quatrième recension et il a fait l'amalgame entre echidna - echidna de mer - sirène, soit nous sommes face à une contamination iconographique de l'echidna-poisson attesté dans le manuscrit de Berne. Le fait d'ailleurs que ce dernier soit un témoin latin n'est pas un obstacle à mon sens. En effet, ce codex, qui est daté du deuxième tiers du IX siècle et qui a été réalisé à Hautvillers (Reims), serait la copie d'un original que plusieurs chercheurs font remonter autour du $\mathrm{IV}^{\mathrm{e}}$ siècle à Alexandrie ${ }^{39}$. C'est un chef d'œuvre de l'enluminure carolingienne avec de fortes réminiscences hellénistiques et, comme le soulignait $\mathrm{V}$. Pakis, ce manuscrit « is thought to derive from a Greek archetype of the type $\Sigma \alpha s »^{40}$. Si cette hypothèse de contamination iconographique s'avérait juste, on serait alors face à une disparité quasi totale entre texte et image dans le Physiologus de Sofia, puisque, répétons-le, l'écrit parle d'un arrière-train en forme de crocodile et l'image lui donne la forme d'une queue de poisson, comme l'echidna de gauche du Bernensis (figs. 1, 5 et 6) ${ }^{41}$. 


\section{Conclusion} étude ne reconnaissent pas la vipère comme étant l'animal auquel renvoie le zoonyme echidna dans le texte du Physiologus, sinon, ils n'auraient pas représenté un être hybride, mais bel et bien un serpent. Leur prise de position est très importante dans la mesure où elle confirme un point de vue clair de la part de ces hommes médiévaux, à des aires chrono-culturelles distinctes et sans contact avéré entre eux, face au zoonyme echidna.

De façon générale, cette mise en regard de l'image et de l'écrit dans les différents manuscrits examinés a permis de tirer quelques informations sur la relation entre ces deux médias. Tout d'abord, on a pu constater que le degré d'inspiration textuelle diffère d'un miniaturiste à l'autre : leurs miniatures ne sont pas subordonnées au verbe, ni aussi figées qu'on le pense souvent. Une véritable complémentarité, mais aussi une réelle concurrence, existent entre ces deux médias. L'exemple de l'echidna étudié ici confirme en effet, une fois de plus, que les rapports entre texte et image sont multiples et souvent difficiles à saisir. Il atteste également de l'indépendance de l'image par rapport au texte et il souligne à quel point les schémas iconographiques peuvent être interchangeables.

Cette mise en regard entre ces deux médias aide aussi à se rendre compte que l'animal dont il est question dans le Physiologus ne peut pas être identifié à la vipère, même si, pour cette dernière, le zoonyme utilisé dans la littérature grecque est le même que celui qu'on trouve dans le Physiologus ${ }^{42}$. L'animal décrit dans cet ouvrage est, clairement, un hybride dont nous ignorons s'il s'agit d'un être imaginé par l'auteur ou si celui-ci se fonde sur des sources inconnues. En outre, le redacteur du Physiologus semble ignorer que les echidnai des passages bibliques qu'il cite dans ce chapitre (Matthieu, 3,7; Luc, 3,7) renvoient à la vipère, sinon comment comprendre la comparaison entre celles-ci et l'echidna, à moins qu'à ses yeux le même zoonyme était un synonyme renvoyant à deux êtres différents.

Soulignons que l'auteur du Phusiologus fait clairement la distinction entre les êtres hybrides, décrits par «moitiés » et le reste des animaux ${ }^{43}$. Remarquons enfin qu'il ne décrit pas l'echidna comme un animal "exotique » distant de son milieu immédiat,

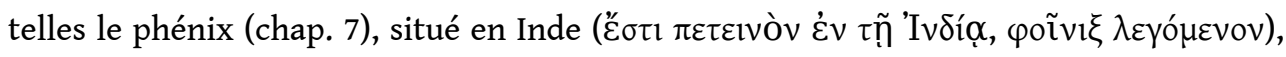

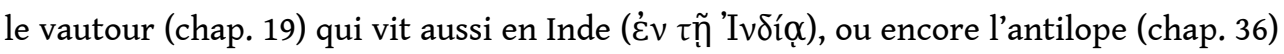

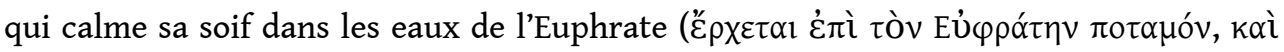

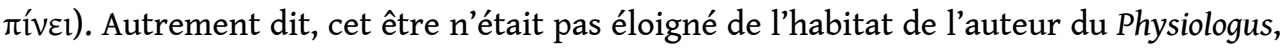
du moins il ne laisse planner aucun doute sur cela. Or, comme tout habitant d'Égypte, il savait bien que la vipère n'est pas un être hybride comme l'échidna qu'il décrit.

De toute façon, les miniaturistes de tous les manuscrits examinés dans la présente chrono-culturelles distinctes et sans contact avéré entre eux, face au zoonyme echidna.

BIBLIOGRAPHIE 


\section{Editions}

Claudius Aelianus, de natura animalium, GARCÍA VALDÉs Manuela , LLERA FUEYo Luis Alfonso , RODRÍGUEZ-NORIEGA GUILLÉN Lucía (éd.), Claudius Aelianus de natura animalium, Berlin, 2009.

Geoponica, ВЕСКH Henricus (éd.), Geoponica sive Cassiani Bassi Scholastici de Re rustica eclogae, Lipsiae, 1895.

Physiologus graecus, GIDEL Charles, LEGRAND Emile (éd.), « Étude sur un poème grec inédit, intitulé ò

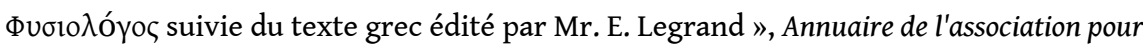
l'encouragement des études grecques en France 7 (1873), p. 188-296.

Physiologus graecus, SBORDONE, Fr. (éd.), Physiologus, Hildesheim - New York, 1936.

Physiologus graecus, offERMANNS, D. (éd.), Der Physiologus nach den Handschriften G und M, Meisenheim am Glan, 1966.

Physiologus, KAIMAKIS, D. (éd.), Der Physiologus nach der ersten Redaktion, Meisenheim am Glan, 1974. Physiologus graecus, STROPPA, M. (éd.), « Un papiro inedito del Fisiologo (PSI inv. 295) », in BASTIANINI G., CASANOVA A. (dir.), Un papiro inedito del Fisiologo (PSI inv. 295), Firenze, 2011, p. 173-192 et tav. XXI-XXII.

Physiologus latinus versio B, CARMODY, Francis J. (éd.), Physiologus latinus : éditions préliminaires versio B, Paris, 1939.

Physiologus latinus versio C, CAHIER, Charles MARTIN, Arthur (éd.), Mélanges d'archéologie, d'histoire et de littérature: collection de mémoires sur l'orfévrerie et les émaux des trésors d'Aix-la-Chapelle, de Cologne, etc., Paris, 1851.

Physiologus latinus versio Y, CARMODY, Francis J. (éd.), Physiologus latinus versio Y, Berkeley - Los Angeles, 1941 (University of California publications in classical philology 12,7).

Skylitzès, Synopsis historiarum, THURN Hans (éd.), Ioannis Scylitzae, Synopsis historiarum, Berlin, 1973.

\section{Travaux}

BAGLIONI Igor, Echidna e i suoi discendenti. Studio sulle entità mostruose della Teogonia esiodea, Roma, 2017.

BERNABÒ Massimo, Il Fisiologo di Smirne: Le miniature del perduto codice B. 8 della Biblioteca della Scuola evangelica di Smirne, Tavarnuzze, 1998.

BODSON Liliane, « Introduction au système de nomination des serpents en grec ancien :

l'ophionyme dipsas et ses synonymes ", Anthropozoologica, 47, 2012, p. 73-155.

BODSON Liliane, «Zoological Knowledge in Ancient Greece and Rome », in CAMPBELL G. L. (dir.), The Oxford Handbook of Animals in Classical Thought and Life, 2014, p. 556-579.

BOUTANTIN Céline, Terres cuites et culte domestique : bestiaire de l'Egypte gréco-romaine, Leiden, 2014.

BRIX Nicole P., Étude de la faune ophidienne de l'Égypte ancienne, Paris, 2006.

DOCAMPo Alvarez P., MARTINEZ Osende J., VILLAR Vidal J., « La version C del Fisiologo latino. El codex Bongarsianus 318 de Berna ", Medievalismo, 10, 2000, p. 27-67.

DOROFEEVA Anna, The reception of the 'Physiologus' in the ninth and tenth centuries, with special reference to Bern, Burgerbibliothek, MS lat. 318, Cambridge, 2011 (mémoire de thèse inédit). 
DRUCE George C., « The Symbolism of the Crocodile in the Middle Ages », Archaeological Journal, 66, 1909, p. 311-338.

DUNAND Françoise, LICHTENBERG Roger (dir.), Des animaux et des hommes : une symbiose égyptienne, Monaco, 2005.

EGGENBERGER Christoph, « Der Physiologus Bernensis. Bild und Text », in KINDSCHI GARSKÝ Z., HIRSCHLUIPOLD R. (dir.), Christus in natura. Quellen, Hermeneutik und Rezeption des Physiologus, Berlin, 2020, p. $189-194$

HOMBURGer Otto, von STEIGER Christoph, Physiologus Bernensis: Voll-Faksimile-Ausgabe des Codex Bongarsianus 318 der Burgerbibliothek Bern, Basel, 1964.

KENNETH Kitchell F., Animals in the ancient world from A to Z, Abingdon, Oxford - New York, 2014. LAZARIS Stavros, Le Physiologus grec, t. 2 : Donner à voir la nature, Firenze [en préparation avancée]. LAZARIS Stavros, « Le Sofija, Naučen Centăr za Slavjano-Vizantijski Proučvanija Ivan Dujčev, D. gr. 297 (olim Kosinitza 244) et ses figures », in Džurova A. (dir.), Le Physiologus de Sofia, Sofia [sous presse].

LAZARIS Stavros, « Manuels d'enseignement dans une bibliothèque monastique du nord de la Grèce : le cas d'un livre illustré d'histoire naturelle et de morale chrétienne », in LEHMANN Y. (dir.), Savoir/Pouvoir. Les bibliothèques, de l'Antiquité à la modernité, Turnhout, 2019, p. 119-138.

LAZARIS Stavros, « Scientific, Medical and Technical Manuscripts », in TSAMAKDA V. (dir.), A Companion to Byzantine illustrated manuscripts, Leiden, 2017, p. 55-113 (et figures p. 3, 8-38).

LAZARIS Stavros, Le Physiologus grec, t. 1 : La réécriture de l'histoire naturelle antique, Firenze, 2016. LAZARIS Stavros, Une œuvre illustrée (pseudo-)scientifique et son lectorat : Le Physiologus grec du Centre Ivan Dujčev (gr. 297), Paris, 2014 (mémoire inédit d'HdR).

LAZARIS Stavros, «Quelques considérations sur l'illustration du Physiologus grec », in VAN DEN ABEELE B. (dir.), Bestiaires médiévaux: Nouvelles perspectives sur les manuscrits et les traditions textuelles. Actes du XVe colloque international de la Société Internationale Renardienne, Louvain-la-Neuve, 18-22 août 2003, Louvain-la-Neuve, 2005, p. 141-167 et figs. 34-50.

LAZARIS Stavros, « Un nouveau manuscrit grec illustré du Physiologus : Au sujet d'une récente étude sur ce texte ", Revue des études byzantines, 58, 2000, p. 279-281.

LECLERCQ-MARX Jacqueline, « La sirène et l'(ono)centaure dans le Physiologus grec et latin et dans quelques bestiaires. Le texte et l'image ", in VAN DEN ABEELE B. (dir.), Bestiaires médiévaux : nouvelles perspectives sur les manuscrits et les traditions textuelles. Actes du XVe colloque de la Société internationale renardienne (Louvain-la-Neuve, 19-22.8.2003), Louvain-la-Neuve, 2005, p. 169-182.

LECLERCQ-MARX Jacqueline, La Sirène dans la pensée et dans l'art de l'antiquité et du Moyen âge : du mythe païen au symbole chrétien, Bruxelles, 1997.

MCCULLOCH Florence, Mediaeval Latin and French bestiaries, Chapel Hill, 1962 (revised edition). MALAXECHEVERRíA Ignacio, « L'Hydre et le crocodile médiévaux », Romance Notes 21 (3), 1981, p. 376-380.

MITTENHUBER Florian, « Die Berner Physiologus-Handschriften. Drei Bücher, drei Geschichten », in KINDSCHI GARSKÝ Z., HIRSCH-LUIPOLD R. (dir.), Christus in natura. Quellen, Hermeneutik und Rezeption des Physiologus, Berlin, 2020, p. 177-188. 
PAKIS Valentine, "Sirens and their Victims in Version B of the Latin 'Physiologus' ", Mittellateinisches Jahrbuch 44 (3), 2009, p. 367-375.

RADOGNA Claudia, « La zoologie médiévale. Le crocodile entre 'historia' et 'ratio' », in CASSIN B., ROMEYER DHERBEY G. (dir.), L'animal dans l'Antiquité, Paris, 1997, p. 519-531.

TRAVIS William J., « Of Sirens and Onocentaurs: A Romanesque Apocalypse at Montceauxl'Etoile », Artibus et Historiae, 23, 45 (2002), p. 29-62.

TSELOS Dimitri, «A Greco-Italian School of Illuminators and Fresco Painters. Its Relation to the Principal Reims Manuscripts and to the Greek Frescoes in Rome and Castelseprio », The art bulletin, 38 (1), 1956, p. 1-30.

VERNUS Pascal, yOYOTTE Jean, Bestiaire des pharaons, Paris, 2005.

VISINTIN Monica, « Di Echidna, e di altre femmine anguiformi », Mètis. Anthropologie des mondes grecs anciens, 12, 1997, p. 205-221.

VOLLENWEIDER Samuel, « Der Erlöser im Tarnanzug. Eine Studie zur Christologie des Physiologus, zu seiner Datierung und zur Rezeptionsgeschichte von Psalm $24\left(23^{\mathrm{LXX}}\right)$ », in KINDSCHI GARSKÝ Z., HIRSCH-LUIPOLD R. (dir.), Christus in natura. Quellen, Hermeneutik und Rezeption des Physiologus, Berlin, 2020, p. 93-132.

WOODRUFF Helen, « The Physiologus of Bern: A Survival of Alexandrian Style in a Ninth Century Manuscript », Art Bulletin 12 (3), 1930, p. 226-253.

ZAMBON Francesco, Bestiari tardoantichi e medievali : $i$ testi fondamentali della zoologia sacra cristiana, Milano, 2018.

ZUCKER Arnaud, Physiologos : Le bestiaire des bestiaires, Grenoble, 2004.

\section{NOTES}

1. Quelques heures avant de déposer cet article, j'apprenais par un mail de V. Pakis le décès de Xénia, survenu le 25 octobre 2019, le jour de son $79^{\text {ème }}$ anniversaire. Que ce petit antidôron, pour toute sa contribution à notre connaissance des manuscrits illustrés du Physiologus, grec et latin, et des bestiaires, lui soit dédié.

2. Sur la date et le lieu de confection du Physiologus grec, voir LAZARIs, Le Physiologus grec, Firenze, 2016 : 17-30. Cette date n'est pas admise par tous les chercheurs, qui penchent parfois pour le III ${ }^{\mathrm{e}}$, voire le IV ${ }^{\mathrm{e}}$ siècle. D'autres l'ont adopté (voir en dernier lieu dans VoLLENWEIDER, "Der Erlöser im Tarnanzug. Eine Studie zur Christologie des Physiologus, zu seiner Datierung und zur Rezeptionsgeschichte von Psalm 24 (23 LXX)", in KINDSCHI GARSKÝ, HIRSCH-LUIPOLD (dir.), Der Erlöser im Tarnanzug. Eine Studie zur Christologie des Physiologus, zu seiner Datierung und zur Rezeptionsgeschichte von Psalm 24 (23 $\left.3^{L X}\right)$, Berlin, 2020 : 93, n. 4).

3. En ce qui concerne la construction des chapitres, d'autres éléments structurels participent à la composition de l'ensemble, dont une citation biblique qu'on trouve au début ou à la fin de plusieurs chapitres. Ces éléments sont vraisemblablement arrangés par l'auteur selon les exigences du thème traité, ce qui explique la grande irrégularité de leur présence dans les chapitres (voir LAZARIS, Le Physiologus, $2016: 84-85$ ).

4. Fr. Sbordone recense 48 chapitres dans son édition critique ( SBORDONE, Physiologus, Hildesheim - New York, 1936 : 1-145). Suite à cette édition, en prenant en compte, entre autres, le New York, Pierpont Morgan Library, Ms. M $397(=$ G), qui constitue le plus ancien témoin complet, D. Offermanns a proposé une nouvelle édition en 49 chapitres des deux premiers sous-groupes de 
cette recension (OFFERMANNS, Der Physiologus nach den Handschriften $G$ und $M$, Meisenheim am Glan, 1966). Quelques années plus tard, D. Kaimakis a publié une édition synoptique des trois autres sous-groupes (KAIMAKIS, Der Physiologus nach der ersten Redaktion, Meisenheim am Glan, 1974). Signalons également l'édition récente d'un papyrus, le PSI inv. 295, du VI siècle, publiée par M. Stroppa (sTROPPA, "Un papiro inedito del Fisiologo (PSI inv. 295)", in BASTIANINI, CASANOVA (dir.), Un papiro inedito del Fisiologo (PSI inv. 295), Firenze, 2011 : 173-192 et tav. XXI-XXII). Il contient une partie des chapitres Sur le bouquetin et Sur la pierre adamantine appartenant à cette première recension.

5. Cf. LAZARIS, Le Physiologus, 2016 : 69-78.

6. Sur ces codex et leur illustration, voir LAZARIS, Le Physiologus grec, Firenze, [en préparation avancée].

7. Sur la discussion concernant la date de copie et de confection des miniatures de ce codex, voir dans BERNABò, Il fisiologo di Smirne: Le miniature del perduto codice B. 8 della Biblioteca della Scuola evangelica di Smirne, Tavarnuzze, 1998.

8. LAZARIS, "Un nouveau manuscrit grec illustré du Physiologus : Au sujet d'une récente étude sur ce texte", Revue des études byzantines, 58 (2000) : 279-281.

9. Sur les mentions isolées dans les différents manuscrits du Physiologus grec, voir SBORDONE (éd.), Physiologus, 1936 : 303-325, ici pp. 316-318.

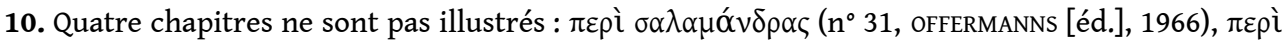

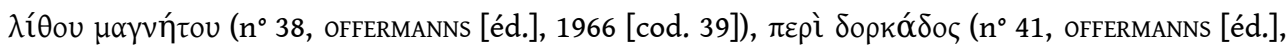

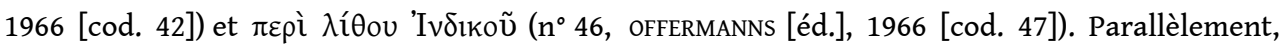
quatre chapitres possèdent plus d'une image, on arrive donc à un total de 55 miniatures. Lesdits chapitres sont les suivants : sur le lion (3 images), sur le serpent (4), sur la fourmi (3), sur les colombes (2).

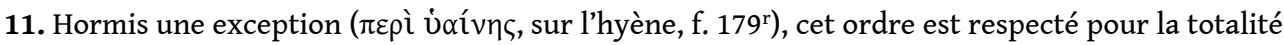
des cas.

12. En effet, excepté une notice publiée l'année qui a suivi la découverte du codex de Sofia (voir supra, n. ${ }^{* * *} 8^{* * *}$ ), j'ai eu l'occasion de lui consacrer deux articles (LAZARIS, "Quelques considérations sur l'illustration du Physiologus grec», in VAN DEN ABEELE (dir.), Quelques considérations sur l'illustration du Physiologus grec, Louvain-la-Neuve, 2005 : 141-167 et figs. 134-150 ; LAZARIS, « Manuels d'enseignement dans une bibliothèque monastique du nord de la Grèce : le cas d'un livre illustré d'histoire naturelle et de morale chrétienne», in LEHMANN, Manuels d'enseignement dans une bibliothèque monastique du nord de la Grèce : le cas d'un livre illustré d'histoire naturelle et de morale chrétienne, Turnhout, 2019 : 119-138), ainsi que le mémoire inédit de mon HdR : Une ceuvre illustrée (pseudo-)scientifique et son lectorat : Le Physiologus grec du Centre Ivan Dujčev (gr. 297), Paris, 2014. Une étude codicologique et paléographique, qui accompagne le fac-similé de ce manuscrit, est par ailleurs sous presse (LAZARIS, «Le Sofija, Naučen Centăr za Slavjano-Vizantijski Proučvanija Ivan Dujčev, D. gr. 297 (olim Kosinitza 244) et ses figures », in A. Džurova (dir.), Le Physiologus de Sofia, Sofia, [sous presse]).

13. Le texte de ce chapitre dans les manuscrits illustrés conservés provient de ces deux recensions. Plusieurs de ces manuscrits se copient l'un l'autre et j'ai décidé d'exclure de cette étude comparative tous ceux qui répètent la même image du point de vue iconographique.

14. Première recension, chap. 10, OFFERMAnNs (éd.), $1966: 46-49$ et KAIMAKIs (éd.), 1974, $n^{\circ} 10$ : 30a-31b ; deuxième recension, chap. 22, SBORDONE (éd.), $1936: 240-242$.

15. Il n'est pas non plus question de "donner à lire » le texte du manuscrit en éliminant des erreurs de copie (le copiste commet notamment des erreurs liées à la phonétique) qui viennent perturber la lecture, y compris l'accentuation et la ponctuation, sauf quand le sens, par rapport au texte de l'édition, est corrompu en profondeur. 
16. Ce témoin contient aux folios $7^{\mathrm{r}}-22^{\mathrm{v}}$ la version $\mathrm{C}$ du Physiologus latin. En plus de cet écrit, il conserve la vie de saint Siméon (ff. $1^{\mathrm{r}}{ }^{\mathrm{r}} \mathrm{r}$ ), la Chronique dite de Frédégaire (ff. $23^{\mathrm{r}}-125^{\mathrm{r}}$ ) et une péricope de l'Évangile de Matthieu avec une traduction latine d'Éphrem le syrien (ff. $125^{\mathrm{v}}-130^{\mathrm{r}}$ ). Le Physiologus latin comporte plusieurs recensions. Les deux plus anciennes sont connues par les sigles $\mathrm{Y}$ et $\mathrm{C}$. La première a été éditée par CARMODY, Physiologus latinus versio $Y$, Berkeley - Los Angeles, 1941. La recension C a été éditée par CAHIER, MARTIN, Mélanges d'archéologie, d'histoire et de littérature : collection de mémoires sur l'orfévrerie et les émaux des trésors d'Aix-la-Chapelle, de Cologne, etc., Paris, 1851. La recension B a été éditée par CARMODY, Physiologus latinus : éditions préliminaires versio $B$, Paris, 1939. Cette recension a eu une grande influence et c'est d'elle que dérivent les principales recensions latines en Angleterre et en France. Sur ce manuscrit, voir, en dernier lieu et avec bibliographie antérieure, MITTENHUBER, «Die Berner Physiologus-Handschriften. Drei Bücher, drei Geschichten », in Kindschi Garský, Hirsch-Luipold (dir.), Die Berner PhysiologusHandschriften. Drei Bücher, drei Geschichten, Berlin, 2020 : 177-188.

17. Il est difficile de déterminer plus en détail cette partie, mais, malgré une certaine ressemblance, il faut exclure un serpentiforme (avec un tube non dégressif) en raison de la présence de la nageoire, ou alors on a affaire à un être composé de trois parties, ce qui ne correspondrait ni au texte du Physiologus, ni à l'iconographie ordinaire de l'echidna.

18. Ed. OFFERMANNS, $1966, \mathrm{n}^{\circ} 10: 46-49$; KAIMAKIS, 1974, $\mathrm{n}^{\circ} 10: 30 \mathrm{a}-31 \mathrm{~b}$.

19. A. Zucker suit la leçon du New York, Pierpont Morgan Library Ms. 397 où il est question de la femelle qui décapite le mâle (ZUCKER, Physiologos, 2004 : 97). Même s'il s'agit du plus ancien témoin de la première recension encore conservé, cette variante me paraît erronée et je reviendrai sur ce point dans une prochaine étude.

20. Trad. ZUCKER, Physiologos: Le bestiaire des bestiaires, Grenoble, 2004 : 97, sauf pour l'identification de cet animal et le passage discutté infra, n. *** $17^{* * *}$.

21. Rien ne permet de mettre en doute l'identification moderne du terme $\varepsilon_{\chi} \downarrow \delta v \alpha$, dans la citation de Matthieu 3.7 et de Luc 3.7, avec la vipère. Cela ne veut pas dire toutefois que l'auteur du Physiologus était conscient de ceci (cf. infra : ${ }^{* * *} 19^{* * *}$ ).

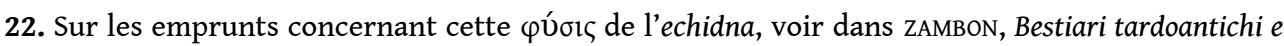
medievali : $i$ testi fondamentali della zoologia sacra cristiana, Milano, $2018: 2352$, n. 15.

23. Les abréviations du scribe sont indiquées entre parenthèses. Le retour à la ligne est affiché par une barre verticale.

24. Certains manuscrits classés par F. Sbordone (SBORDONE, 1936 : xviii-xxv) dans la deuxième recension, puisent leur texte depuis la première et la deuxième (voire pour quelques rares cas, depuis la troisième) recension, d'où ma décision de les classer dans une catégorie à part. Pour plus de détail et un classement plus subtil, voir LAZARIS, Le Physiologus [en préparation avancée].

25. Tel est le cas par exemple du Leipzig, Universitätsbibliothek, cod. gr. 35.

26. Le corps/queue de cette partie forme des spires, typiques non seulement des ophidés, mais également des crocodiles (voir plusieurs exemples dans DRUCE, « The Symbolism of the Crocodile in the Middle Ages », Archaeological Journal, 66 (1909) : 311-338).

27. Voir dans MCCULloch, Mediaeval Latin and French bestiaries, Chapel Hill, 1962 (Revised edition) : 24.

28. Ce n'est pas une queue de poisson ordinaire, car les poissons, dans l'art antique et médiéval, ne sont pas représentés habituellement avec une queue à torsades. Hormis Triton, de telles queues sont reprises pour plusieurs figures médiévales de la sirène (voir quelques exemples dans LECLERCQ-MARX, La Sirène dans la pensée et dans l'art de l'antiquité et du Moyen âge : du mythe païen au symbole chrétien, Bruxelles, 1997).

29. En Grèce classique la sirène est figurée sous forme de monstre mi-femme, mi-oiseau (voir p. ex. une terre cuite produite vers $450-425$ av. J.-C. et conservée au musée du Louvre [ ${ }^{\circ}$ inv. MNB1731] représentant une sirène béotienne coiffée d'un polos à trois pointes). Peu à peu, elle 
sera pourvue de bras et d'une poitrine. Puis, sous l'influence des mythologies celtique et germanique, elle perdra ses caractéristiques aviaires pour se transformer en être pisciforme. Sur la sirène dans l'art et la littérature au Moyen Âge, voir LECLERCQ-MARX, La Sirène dans la pensée, 1997 ; TRAVIS, « Of Sirens and Onocentaurs: A Romanesque Apocalypse at Montceaux-l'Etoile ", Artibus et Historiae, 23, 45 (2002) : 29-62 ; LECLERCQ-MARX, « La sirène et l'(ono)centaure dans le Physiologus grec et latin et dans quelques bestiaires. Le texte et l'image ", in VAN DEN ABEELE (dir.), La sirène et l'(ono)centaure dans le Physiologus grec et latin et dans quelques bestiaires. Le texte et l'image, Louvainla-Neuve, $2005: 169-182$; PAKIS, "Sirens and their Victims in Version B of the Latin Physiologus ", Mittellateinisches Jahrbuch 44,3 (2009) : 367-375.

30. Le haut du corps de celle-ci est celui d'une belle jeune fille, le bas celui d'un serpent redoutable qui vit sous terre, au pays des Arimes, dans un antre situé loin du regard des dieux et des hommes. Sur l'Echidna, cf. VISINTIN, «Di Echidna, e di altre femmine anguiformi », Mètis. Anthropologie des mondes grecs anciens, 12 (1997) : 205-221 ; BAGLIONI, Echidna e i suoi discendenti. Studio sulle entità mostruose della Teogonia esiodea, Roma, 2017.

31. Partout il est précisé que le corps inférieur de l'echidna est semblable à celui d'un crocodile. Ce chapitre apparait dans les quatre recensions grecques, mais seule la première, deuxième et quatrième donnent la description de cet être.

32. Dans le manuscrit de Milan par exemple, le crocodile est représenté de façon naturaliste (f. $26^{\mathrm{r}}$.

33. Cf. DRUCE, "The Symbolism of the Crocodile in the Middle Ages ", Archaeological Journal, 66 (1909) : 311-338 ; MALAXECHEVERRíA, «L'Hydre et le crocodile médiévaux », Romance Notes, 21, 3 (1981) : 376-380 ; RADOGNA, «La zoologie médiévale. Le crocodile entre 'historia' et 'ratio' », in CASSIN, ROMEYER DHERBEY (dir.), La zoologie médiévale. Le crocodile entre 'historia' et 'ratio', Paris, 1997 : 519-531.

34. Par ailleurs, si l'explication de l'assimilation entre crocodile et serpent au Moyen Âge permet de comprendre la représentation du serpent au lieu du crocodile dans les manuscrits médiévaux grecs (sauf dans celui de Sofia), elle n'est que très difficilement acceptable pour le texte du Physiologus. Rappelons en effet que son auteur a vécu en Égypte, plus précisément à Alexandrie, autour du II ${ }^{\mathrm{e}}$ siècle, et connaissait donc parfaitement aussi bien le crocodile que le serpent. Il n'y a en effet pas assimilation en Égypte entre ces deux animaux, sauf le cas de la déesse-serpent Renenoutet, étroitement alliée au dieu-crocodile Sobek (cf. DUNAND, LICHTENBERG, Des animaux et des hommes : une symbiose égyptienne, Monaco, 2005 : 138-139). Sur ces deux animaux en Égypte, voir VERNUS, YOYOTTE, Bestiaire des pharaons, Paris, 2005 ; BRIX, Étude de la faune ophidienne de l'Égypte ancienne, Paris, 2006 ; BOUTANTIN, Terres cuites et culte domestique : bestiaire de l'Egypte greco-romaine, Leiden, 2014. Je tiens à remercier Antonio Ricciardetto pour ces informations. Si donc cette explication pouvait éclairer l'iconographie choisie par les miniaturistes médiévaux, cela ne justifie aucunement la raison pour laquelle le rédacteur du Physiologus a préféré le crocodile plutôt que le serpent dans la description morphologique de l'echidna.

35. Exception faite du registre supérieur dans le Vat. Ottob. gr. 354 et des créatures de gauche et de droite dans le Vindob. Phil. gr. 290.

36. Il est notamment écrit que l'echidna de mer s'attache à un navire, paralyse les mouvements du

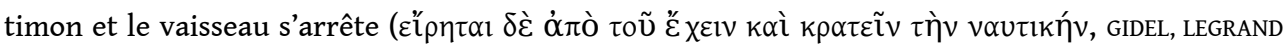

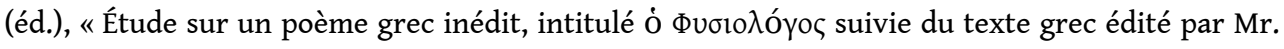
E. Legrand ", Annuaire de l'association pour l'encouragement des études grecques en France, 7 (1873) : 188-296, ici pp. 247-248).

37. Transcription du texte et traduction allemande : HOMBURGER, STEIGER, Physiologus Bernensis: VollFaksimile-Ausgabe des Codex Bongarsianus 318 der Burgerbibliothek Bern, Basel, 1964 : 70-71. 
38. Sur ce manuscrit qui est dorénavant daté vers 1060, voir LAZARIS, «Scientific, Medical and Technical Manuscripts », in TSAMAKDA (dir.), Scientific, Medical and Technical Manuscripts, Leiden, 2017 : 55-113 et figs. $113,118-138$, ici p. 81.

39. Sur le Physiologus de Berne, hormis les références citées supra, voir WOODRUFF, "The Physiologus of Bern: A Survival of Alexandrian Style in a Ninth Century Manuscript ", Art Bulletin, 12,3 (1930) : 226-253 ; TSELOS, «A Greco-Italian School of Illuminators and Fresco Painters. Its Relation to the Principal Reims Manuscripts and to the Greek Frescoes in Rome and Castelseprio ", The art bulletin, (1956) : 1-30; DOCAMPO ALVAREZ, MARTINEZ OSENDE, VILLAR VIDAL, «La version C del Fisiologo latino. El codex Bongarsianus 318 de Berna », Medievalismo, 10 (2000) : 27-67; Dorofeeva, The reception of the 'Physiologus' in the ninth and tenth centuries, with special reference to Bern, Burgerbibliothek, MS lat. 318, Cambridge, 2011 (mémoire de these inédit) ; EGGENBERGER, «Der Physiologus Bernensis. Bild und Text», in Kindschi Garský, Hirsch-Luipold (éd.), Der Physiologus Bernensis. Bild und Text, Berlin, 2020 : 189-194.

40. PAKIS, « Sirens and their Victims », $2009: 367$.

41. Dans le Physiologus de Vienne (deux hybrides de gauche), nous remarquons aussi un rapport extrêmement étroit avec le texte. Sur les raisons de cette discordance, voir LAZARIs, Le Physiologus [en préparation avancée].

42. Voir KENNETH, Animals in the ancient world from A to Z, Abingdon, Oxon - New York, NY, 2014 : 63-64. En plus des références citées dans cet ouvrage, on peut signaler un passage dans les Geoponica (15.1.16, éd. BECKH, Geoponica sive Cassiani Bassi Scholastici de Re rustica eclogae, Lipsiae, 1895 ) et dans le Skylitzès (Synopsis historiarum, Mich 4.4.19, éd. Ioannis Scylitzae Synopsis historiarum, ed. THURN, Berlin, 1973). Sur le zoonyme echidna et son identification avec la vipère, voir également BODSON, «Introduction au système de nomination des serpents en grec ancien : l'ophionyme dipsas et ses synonymes ", Anthropozoologica, 47 (2012) : 73-155, ici p. 78-79 ; BoDson, «Zoological Knowledge in Ancient Greece and Rome », in Campbell (dir.), Zoological Knowledge in Ancient Greece and Rome, 2014 : 556-579, ici p. 563. En dehors de la littérature, dans plusieurs manuscrits grecs illustrés, le zoonyme echidna est compris par les miniaturistes comme étant un serpent (voir à titre d'exemple dans le Paris, BNF, gr. 2183, ff. $35^{\mathrm{r}}$ et $159^{\mathrm{v}}$ et dans le Paris, BNF, Suppl. gr. 247, ff. $\left.2^{\mathrm{v}}, 6^{\mathrm{r}}, 7^{\mathrm{r}}, 9^{\mathrm{v}}\right)$.

43. Voir dans LAZARIS, Le Physiologus, $2016: 83-84$.

\section{RÉSUMÉS}

La présente étude se penche sur les représentations de l'echidna dans les manuscrits illustrés du Physiologus; plus précisément, elle s'intéresse à une variante iconographique relevée dans le Physiologus de Sofia (Dujčev gr. 297). La comparaison avec les autres codex illustrés permet ainsi de révéler certains particularismes de ce témoin, très important pour notre connaissance de l'iconographie de cette œuvre. Par ailleurs, en s'intéressant aux rapports entre le texte et l'image dans les différents manuscrits examinés et les renseignements que l'on peut en tirer pour l'identification de l'echidna, cet article souligne l'importance de l'iconographie. Celle-ci permet, dans l'exemple précis, de corriger une erreur d'identification moderne. Il ne faut, en effet, pas oublier que ces images sont le témoignage direct de la façon dont les hommes et les femmes de leur époque comprenaient tel ou tel zoonyme, en suivant les explications de l'auteur de 
l'ouvrage, des informations dans d'autres textes ou encore depuis des sources pas toujours évidentes à saisir par nous.

This study examines echidna representations in the illustrated manuscripts of the Physiologus. In this contribution, I was specifically interested in an iconographic variant found in the Sofia Physiologus (Dujčev gr. 297). Comparison with other illustrated codices revealed certain particularities of this source, very important for our iconographic knowledge of this work. In addition, by focusing on the relationship between text and image in the various manuscripts under scrutiny and the information that can be obtained from them with regards to the identification of the echidna, I wanted to emphasize the importance of iconography. In this specific example, it helped to correct a modern identification error. It should not be forgotten that these images are a direct testimony to the way in which men and women of the past understood this or that zoonym in their era, following the explanations of the author of the work, information in other texts or even from sources that are not always obvious to us.

\section{INDEX}

Mots-clés : Echidna, vipère, rapports texte-image, Physiologus, Élien, Nicandre, Pline

Keywords : Echidna, Viper, Text-Picture Relation, Physiologus, Aelian, Nicander, Plinius

\section{AUTEUR}

\section{STAVROS LAZARIS}

Stavros Lazaris est chargé de recherche au CNRS et habilité à diriger des recherches par l'EPHE. Ses recherches et son enseignement concernent l'histoire de la civilisation byzantine, notamment à travers l'étude de documents originaux relatifs à l'histoire des sciences et des techniques et de l'histoire des textes et des images. Il est l'auteur de nombreux articles vel ouvrages sur la zoologie byzantine (en particulier les équidés) et l'iconographie médiévale. 